OPEN ACCESS

Edited by:

Kin-Ming (Clement) Tsui, Weill Cornell Medicine - Qatar, Qatar

Reviewed by:

Hamid Badali,

Mazandaran University of Medical Sciences, Iran

Peiying Feng,

Sun Yat-sen University, China

*Correspondence:

Popchai Ngamskulrungroj popchai.nga@mahidol.ac.th

Specialty section: This article was submitted to Fungi and Their Interactions,

a section of the journa

Frontiers in Microbiology

Received: 03 January 2019 Accepted: 13 May 2019

Published: 14 June 2019

Citation:

Pham LTT, Pharkjaksu S, Chongtrakool P, Suwannakarn $K$ and

Ngamskulrungroj $P$ (2019) A Predominance of Clade 17 Candida albicans Isolated From Hemocultures in a Tertiary Care Hospital in Thailand.

Front. Microbiol. 10:1194. doi: 10.3389/fmicb.2019.01194

\section{A Predominance of Clade 17 Candida albicans Isolated From Hemocultures in a Tertiary Care Hospital in Thailand}

\author{
Linh Thi Truc Pham, Sujiraphong Pharkjaksu, Piriyaporn Chongtrakool, \\ Kamol Suwannakarn and Popchai Ngamskulrungroj*
}

Department of Microbiology, Faculty of Medicine Siriraj Hospital, Mahidol University, Bangkok Noi, Thailand

Candida albicans is one of the most common human fungal pathogens. Candidemia has significant mortality globally. No epidemiological study of $C$. albicans based on multilocus sequence typing (MLST) has been conducted in Thailand. Therefore, MLST was used to study the molecular epidemiology of $C$. albicans blood strains in a large Thai teaching hospital. In vitro virulence phenotypes and antifungal susceptibility testing by broth microdilution were also conducted. Forty-six C. albicans blood strains from 37 patients were collected from the Department of Microbiology, Siriraj Hospital, in 2016 and 2017 . Most patients (71.8\%) were more than 60 years old, and the case fatality rate was $54.8 \%$. The male-to-female ratio was 5:3. Thirty-four diploid sequence types (DSTs), including six new DSTs, were identified, with DST2514 (8.7\%) and DST2876 $(8.7 \%)$ as the most common DSTs. Strains were clustered into nine clades. Unlike other studies of $C$. albicans blood strains in Asia, clade 17 was the most common (13 strains, 28.3\%). Sequential allelic changes were evident in sequential strains from one patient. All strains produced phospholipase and hemolysin, while none produced proteinase. The ability to form biofilm was found in $82.6 \%$ of the strains. Clade 17 strains showed significantly stronger hemolytic activity than non-clade 17 strains $(69.2 \%$ versus $27.3 \%$; $p=0.022$ ). However, no significant association existed between clades and patient mortalities. All were susceptible or wild type to anidulafungin ( $\mathrm{MIC}$ range $=0.015-$ 0.12 and $\mathrm{GM}=0.030$ ), micafungin ( $\mathrm{MIC}$ range $=\leq 0.008-0.015$ and $\mathrm{GM}=0.008$ ), caspofungin ( $\mathrm{MIC}$ range $=0.008-0.12$ and $\mathrm{GM}=0.036$ ), and amphotericin $\mathrm{B}$ (MIC range $=0.25-0.5$ and $\mathrm{GM}=0.381)$. Only one strain was resistant to voriconazole ( $\mathrm{MIC}$ range $=\leq 0.008$ to $\geq 8$ and $\mathrm{GM}=0.010)$ and fluconazole $(\mathrm{MIC}$ range $=0.12$ 16 and $\mathrm{GM}=0.398$ ). In conclusion, a high prevalence of clade $17 \mathrm{C}$. albicans blood strains was found in Thailand, in contrast to other Asian countries. This unique finding might be explained by the strong hemolytic activity that is required for bloodstream infection of $C$. albicans.

Keywords: Candida albicans, clade, multilocus sequence typing, virulence factors, antifungal susceptibility, candidemia 


\section{INTRODUCTION}

Candida albicans is a member of the human flora and is commonly colonized in the human digestive tract. C. albicans is an important cause of candidiasis (Bougnoux et al., 2006). It can cause infections that range from superficial infections to life-threatening systemic infections. Approximately $75 \%$ of all women suffer from vulvovaginal candidiasis at one point in their life span, and C. albicans accounts for nearly $90 \%$ of such cases (Yazdanparast et al., 2015). C. albicans and other Candida species including C. glabrata, C. parapsilosis, C. tropicalis, and C. krusei can travel through the bloodstream and together are causative agents in $90 \%$ of invasive candidiasis cases (Vaezi et al., 2017). Many risk factors contributing to candidiasis were reported, including host-related factors such as immunosuppressive disorders, neutropenia, age, or debilitating diseases, and healthcare-associated factors such as catheter use, surgical interventions, transplantation, or the use of antimicrobial drugs (Yapar, 2014; Diba et al., 2018).

Virulence is defined as the ability of a microbe to damage its host (Casadevall and Pirofski, 1999), and C. albicans possesses a wide range of virulence factors, including phenotype switching, adhesin expression, thigmotropism, biofilm formation, and extracellular hydrolytic enzyme secretion, to facilitate tissue invasion and ultimately produce the diseases (Mayer et al., 2013). A recent trend showed increased resistance of non-albicans Candida to azole-based antifungal agents, echinocandins, and multiple classes of drugs (Wiederhold, 2017). However, C. albicans remains highly susceptible to most antifungal drugs (Bustamante et al., 2014; Posteraro et al., 2015; Doi et al., 2016; Tadec et al., 2016; Tan et al., 2016; Chapman et al., 2017).

Several genotyping methods have been used to study the molecular epidemiology of C. albicans. Multilocus sequence typing (MLST) has been widely used to study the molecular characterization of $C$. albicans based on the sequences of seven housekeeping genes (AAT1a, ACC1, ADP1, MPI1b, SYA1, VPS13, and ZWF1b) (Bougnoux et al., 2003; Afsarian et al., 2015). This method has identified 19 clades worldwide (Shin et al., 2011; Gong et al., 2018). A 2007 global study of 1,391 C. albicans strains showed that clade 1 was the most common (33.6\%) (Odds et al., 2007). In Asia, the second and third most common clades were clades $4(8.8 \%)$ and 17 (8.2\%) (Odds et al., 2007). When considering only blood strains, a 2016 Korean study of 149 C. albicans blood strains showed that clade 18 was the most common (18.8\%), followed by clade $4(15.4 \%)$ and clade $1(14.8 \%)$.

So far, no epidemiological study of C. albicans based on MLST has been conducted in Thailand. Therefore, MLST was used to study the molecular epidemiology of $C$. albicans strains of candidemia patients at Siriraj Hospital, including virulence factors and antifungal susceptibility profiles.

\section{MATERIALS AND METHODS}

\section{Study Sites and Subject Selection}

All C. albicans strains were collected from positive hemocultures from a diagnostic microbiology laboratory of the Department of Microbiology, Siriraj Hospital, between April 2016 and November 2017. This study was approved by the Siriraj Institutional Review Board, no. SI 091/2016. Informed consent was not required in this work. All strains, collected from the same patients, were considered sequentially collected, as blood samples were taken from different body sites at different time points. Forty-six strains from 37 individual candidemia patients were collected. Available patient records were retrieved from the hospital registry. A healthcare-associated infection was defined as an onset of candidemia symptoms and/or signs after $48 \mathrm{~h}$ of admission. As the hospital is a tertiary care referral center, patient origins varied. Most cases (81.25\%; 26 of the 32 available patient records) were from central Thailand; 6.25, 6.25, and $6.25 \%$ of cases were from Eastern, Northeastern, and Western Thailand, respectively. The case fatality rate was calculated as the ratio of patient deaths to all candidemia cases in this study. All strains were identified using CHROMagar Candida chromogenic media (Oxoid, United Kingdom) and RapID ${ }^{\mathrm{TM}}$ YEAST PLUS System (Thermo Scientific, United States) followed by singlecolony subculture on Sabouraud dextrose agar (SDA; Oxoid, United Kingdom) to prepare pure cultures before performing any further analysis.

\section{MLST Analysis}

The seven housekeeping genes including AAT1a, ACC1, ADP1, $M P I b, S Y A 1, V P S 13$, and ZWF1b were used for MLST according to a previously published method (Bougnoux et al., 2002). Briefly, PCR mixtures were prepared in $50 \mu 1$ reaction volumes containing DNase-RNase free water, $10 \times$ buffer, $25 \mathrm{mM} \mathrm{MgCl}_{2}$, $2.5 \mathrm{mM}$ dNTPs, $10 \mu \mathrm{M}$ for each of the forward and reverse primers of each gene, $0.25 \mu \mathrm{l}$ of $5 \mathrm{U} / \mu \mathrm{l}$ Taq DNA polymerase (Thermo Scientific, United States), and genomic DNA. The condition was set up with a denaturation step at $95^{\circ} \mathrm{C}$ for $5 \mathrm{~min}$, followed by 35 cycles of $95^{\circ} \mathrm{C}$ for $40 \mathrm{~s}$, annealing at $50^{\circ} \mathrm{C}$ for $40 \mathrm{~s}$, extension at $72^{\circ} \mathrm{C}$ for $1 \mathrm{~min}$, and a final extension step at $72^{\circ} \mathrm{C}$ for $4 \mathrm{~min}$. Approximately $50 \mathrm{ng}$ of DNA was sequenced using each forward and reverse primer on a DNA analyzer (First Base Company, Singapore). The generated sequences were manually edited using MEGA6 software and aligned using Clustal W. The allelic profile (allele number) for each gene and allele combination (diploid sequence type, DST) for the seven loci of each isolate was assigned, or new DST numbers were obtained from the C. albicans MLST database ${ }^{1}$. For cluster analysis, because the base at each polymorphic site can be homozygous or heterozygous, the nucleotide bases were manually edited according to a previous publication (Tavanti et al., 2005). The bases of each taxon were doubled when the site was homozygous and written in pairs when the site was heterozygous. Relationships among concatenated sequences of the seven loci of each strain were determined using cluster analysis using the unweighted pair group method using their arithmetic averages (UPGMA) and the neighbor joining (NJ) method determined by p distance with pairwise deletion of the MEGA 6 software. A bootstrap of 1,000 replications was used for the construction (Shin et al., 2011; Wu et al., 2017). Bootstrap

\footnotetext{
${ }^{1}$ https://pubmlst.org/calbicans/
} 
values of $\geq 75 \%$ were defined as statistically significant. Finally, clade numbers were assigned according to previous publications (Odds et al., 2007; Tsai et al., 2015; Wu et al., 2015; Gong et al., 2018) or by using the eBURST method as described previously (Odds et al., 2007). DSTs from clade numbers that were not presented in our strains were also included as reference DSTs for UPGMA and NJ analysis (Figure 1).

\section{In vitro Virulence Study}

The test for C. albicans phospholipase activity was performed on egg yolk medium according to a previous study

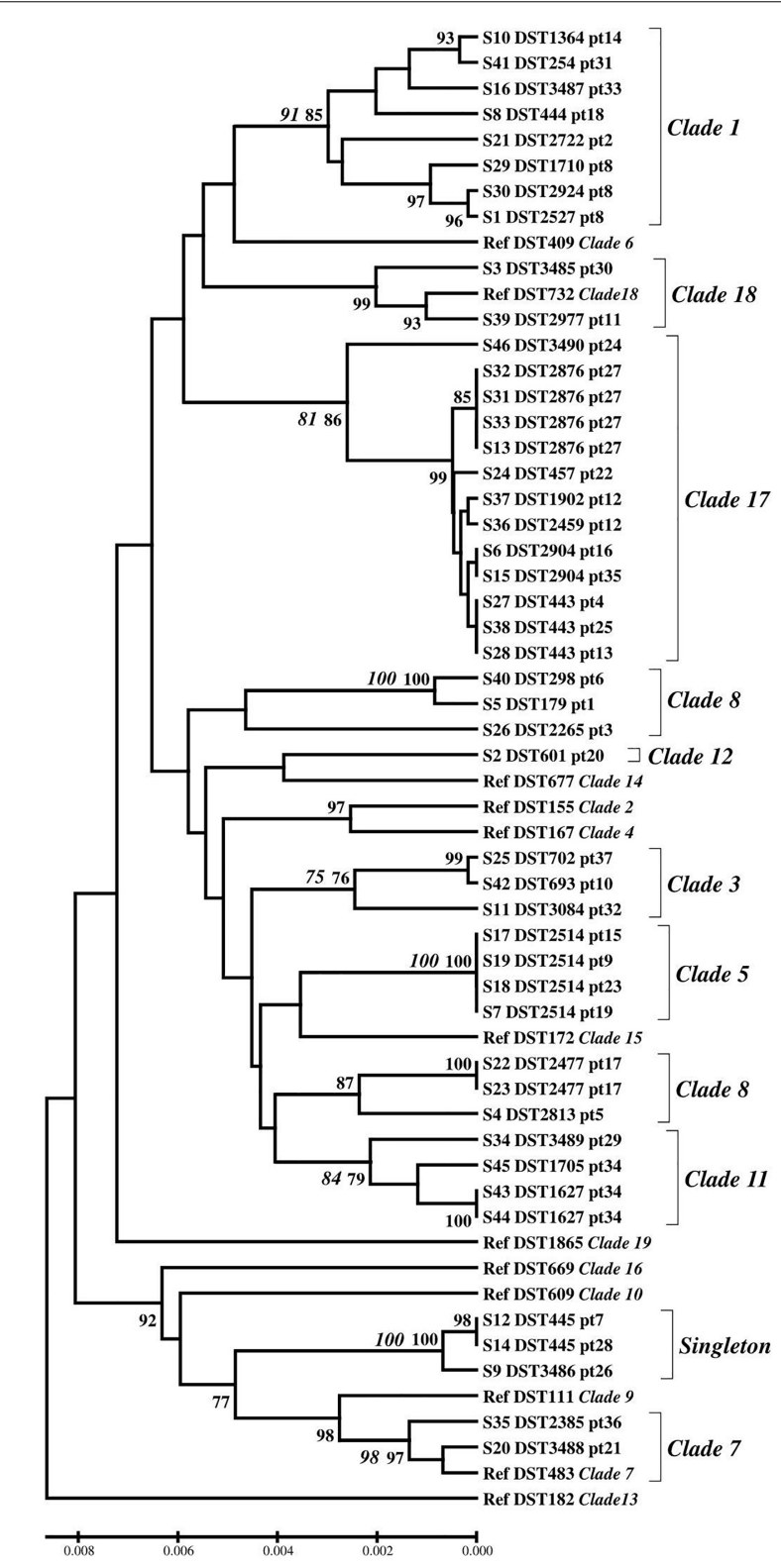

FIGURE 1 | Genetic diversity inferred by UPGMA. Bootstrap support values above 75 for UPGMA (bold) and NJ method (italic bold) were indicated. Ref; reference DST, pt; patient number.
(Price et al., 1982). Strains were grown on SDA for $24 \mathrm{~h}$ before adjusting to a $0.5 \mathrm{McFarland}$ standard $\left(10^{8}\right.$ cells $\left./ \mathrm{ml}\right)$. One hundred milliliters of medium containing $13.0 \mathrm{~g}$ SDA, $11.7 \mathrm{~g} \mathrm{NaCl}$ (Merck, Germany) SDA, $11.7 \mathrm{~g} \mathrm{NaCl}$ (Merck, Germany), $1.48 \mathrm{~g} \mathrm{CaCl}_{2} \cdot 2 \mathrm{H}_{2} \mathrm{O}$ (Merck, Germany), 2\% agar (Oxoid, United Kingdom), and 10\% sterile egg yolk (Merck, Germany) was prepared to determine extracellular phospholipase activity (Tsang et al., 2007). Five microliters of a yeast suspension $\left(10^{8}\right.$ cells $\left./ \mathrm{ml}\right)$ of the tested and control strains was dropped onto the surface of the egg yolk-containing plate and incubated at $37^{\circ} \mathrm{C}$ for $48 \mathrm{~h}$ to measure a precipitation zone around the colony. Extracellular phospholipase activity of $C$. albicans was considered positive when a white precipitation zone was visible around the colony on the plate (Macfarlane and Knight, 1941).

Candida albicans strains' proteinase activity was analyzed on bovine serum albumin medium by the modified Staib method (Staib, 1965; Sachin et al., 2012). Five microliters of a yeast suspension $\left(10^{8}\right.$ cells $\left./ \mathrm{ml}\right)$ of the tested and control strains was dropped on a medium containing $1.17 \%$ yeast carbon base (Becton Dickinson, United States), 0.2\% bovine serum albumin (GoldBio, United States), 2\% agar (Oxoid, United Kingdom), and $0.01 \%$ yeast extract (HiMedia, India) and then incubated at $37^{\circ} \mathrm{C}$ for 2 days. The presence of a clear halo zone around the colony was recorded as evidence of proteinase activity.

To measure hemolytic activity, a medium was prepared by adding $7 \mathrm{ml}$ fresh sheep blood (Clinag, Thailand) to $100 \mathrm{ml}$ SDA supplemented with $3 \%$ of enriched glucose (HiMedia, India) (Luo et al., 2001). Five microliters of a yeast suspension $\left(10^{8}\right.$ cells $/ \mathrm{ml}$ ) of the tested and control strains was spotted onto the medium. The plate was then incubated at $37^{\circ} \mathrm{C}$ for $48 \mathrm{~h}$. The presence of a distinct translucent halo indicates positive hemolytic activity.

The ratios of the diameter of the yeast colony to the total diameter of the colony plus the white precipitation zone, the clear halo zone, and the translucent halo zone were used to represent the level of phospholipase, proteinase, and hemolytic activity, respectively (Sachin et al., 2012).

Biofilm formation was determined using the 2,3bis(2-methoxy-4-nitro-5-sulfophenyl)-2H-tetrazolium-5-carbox anilide (XTT; Thermo Scientific, United States) reduction assay using 96-well microplates as indicated in previous studies (Yigit et al., 2011; Oz et al., 2012). Briefly, yeast cells were adjusted to a $0.5 \mathrm{McFarland}$ standard with yeast extract-peptone-dextrose medium. One hundred microliters of yeast suspension was seeded in 96 -well plates and incubated at $37^{\circ} \mathrm{C}$ for $48 \mathrm{~h}$. The cells were then washed twice with phosphate-buffered saline to remove non-adhering cells. A 50- $\mu \mathrm{l}$ mixture of $1 \mathrm{mg} / \mathrm{ml}$ XTT and $10 \mu \mathrm{l}$ of activation reagent, phenazine methosulfate (HiMedia, India), was added to the wells and further incubated in the dark for $2 \mathrm{~h}$. The biofilms were quantified at $\mathrm{A}_{490}$ and defined as low biofilm formation and high biofilm formation by comparing the XTT activity of each strain to the geometrical mean as described previously (Li et al., 2003; Hasan et al., 2009).

Each tested virulence phenotype's expression levels were classified according to their reference methods (Price et al., 1982; 
Kantarcioglu and Yucel, 2002; Li et al., 2003; Tsang et al., 2007; Hasan et al., 2009) as follows: zone of activity of phospholipase and proteinase (Price et al., 1982; Kantarcioglu and Yucel, 2002; Tsang et al., 2007): $\leq 0.69=$ very strong, $0.70-0.79=$ strong, 0.80-0.89 = medium, $0.90-0.99=$ weak, and $1.00=$ negative; hemolytic activity (Rossoni et al., 2013): $<0.64=$ strong positive, $0.64 \leq$ to $<1=$ positive, and $1.00=$ negative; and biofilm formation (Li et al., 2003; Hasan et al., 2009): O.D. value > GM (geometric mean) = high biofilm formation, O.D. value $\leq \mathrm{GM}=$ low biofilm formation, and O.D. value $<0.10=$ negative biofilm formation .

\section{Antifungal Susceptibility Testing}

Antifungal susceptibility testing of the $C$. albicans strains was conducted using a commercial microdilution method, Sensititre ${ }^{\circledR}$ YeastOne $^{\circledR}$ part YO10 (SYO; Thermo Scientific, United States). The concentrations of antifungal drugs were: anidulafungin $0.015-8 \mu \mathrm{g} / \mathrm{ml}$, micafungin $0.008-$ $8 \mu \mathrm{g} / \mathrm{ml}$, caspofungin $0.008-8 \mu \mathrm{g} / \mathrm{ml}$, amphotericin B 0.12-8 $\mu \mathrm{g} / \mathrm{ml}$, 5-fluorocytosine $0.06-64 \mu \mathrm{g} / \mathrm{ml}$, posaconazole $0.008-8 \mu \mathrm{g} / \mathrm{ml}$, voriconazole $0.008-8 \mu \mathrm{g} / \mathrm{ml}$, itraconazole $0.015-16 \mu \mathrm{g} / \mathrm{ml}$, and fluconazole $0.12-256 \mu \mathrm{g} / \mathrm{ml}$, according to the manufacturer's protocol. C. krusei ATCC 6258 and C. parapsilosis ATCC 22019 were used as reference strains. Briefly, $20 \mu \mathrm{l}$ of yeast suspension McFarland standard no. 0.5 (approximately $10^{6}$ cells $/ \mathrm{ml}$ ) was suspended in $11 \mathrm{ml}$ of YeastOne ${ }^{\circledR}$ inoculum broth to give a final working yeast suspension of approximately $1.5-2.5 \times 10^{3}$ $\mathrm{cfu} / \mathrm{ml}$. Then, $100 \mu \mathrm{l}$ of working yeast suspension was added to each well using a multichannel pipetting device. The SYO panels were covered with adhesive seals and incubated at $35^{\circ} \mathrm{C}$ for $24 \mathrm{~h}$. If the positive control showed no yeast growth, the plate was incubated for an additional $24 \mathrm{~h}$. Minimum inhibitory concentration (MIC) results for all test agents were read according to the manufacturer's instructions. The criteria used to interpret the epidemiological cutoff values (ECVs) and clinical break points (CBPs) were based on Clinical and Laboratory Standards Institute (CLSI) guidelines: ECVs of anidulafungin $=0.12$, micafungin $=0.03$, and amphotericin $\mathrm{B}=2 ; \mathrm{CBPs}$ of anidulafungin, micafungin, and caspofungin: $S \leq 0.25$, $\mathrm{I}=0.5, \mathrm{R} \geq 1$; voriconazole: $\mathrm{S} \leq 0.12, \mathrm{SDD}=0.25-$ $0.5, \mathrm{R} \geq 1$; and fluconazole: $\mathrm{S} \leq 2, \mathrm{SDD}=4, \mathrm{R} \geq 8$ (Clinical Laboratory Standards Institute, 2012, 2016).

\section{Statistical Analysis}

PASW Statistic 18 (IBM Corporation Armonk, NY, United States) was used for data analysis. To estimate frequencies, descriptive statistical tools were used. GM for biofilm formation was calculated using Microsoft Excel 2013 (Microsoft Corporation, Redmond, WA, United States). Statistical significance of an association between the MLST clusters and virulence phenotype was determined using chi-square or Fisher's exact test (PASW version 18, IBM, Bangkok, Thailand); $p \leq 0.05$ was considered statistically significant.

\section{RESULTS}

\section{Most Candidemia Cases Occurred as Hospital-Associated Infections and in Older Patients}

Clinical data were available from 32 of the 37 patients. All patients (100\%) had one or more immunocompromised conditions, including receiving immunosuppressants or chemotherapy (10 patients, $31.3 \%$ ), having diabetes mellitus (10 patients, $31.3 \%$ ), being a newborn ( 2 patients, $6.2 \%$ ), and old age ( $>60$ years old) (23 patients, $71.8 \%$ ). The case fatality rate was $54.8 \%$. The maleto-female ratio was 5:3 (Supplementary Table S1). Most cases (31 patients, 96.9\%) were hospital-associated infections except one who had Candida sepsis as a primary diagnosis. A primary source of candidemia was not found in patients in this study.

\section{MLST Clade 17 and Clade 1 Were Predominant in C. albicans Blood Strains in Thailand}

The most common allele types of the AAT1a, ACC1, ADP1, $M P I b, S Y A 1, V P S 13$, and ZWF1b genes were allele types 59 (26.1\%), 5 (28.3\%), 21 (39.1\%), 2 (30.4\%), 80 (23.9\%), 108 (19.6\%), and 15 (32.6\%), respectively (Supplementary Table S1). The 46 strains yielded 34 distinct DSTs. Forty strains (86.9\%) belonged to previously described types (28 DSTs), whereas the remaining six strains (13.0\%) belonged to novel DSTs (DST3485, DST3486, DST3487, DST3488, DST3489, and DST3490) that had not previously been identified (Supplementary Table S1). Among the 34 DSTs, the most common DSTs were DST2514 and DST2876 (8.7\% for each DST), followed by DST443 (6.5\%) and DST1627, DST2477, DST2904, and DST445 (4.4\% for each DST). Based on the UPGMA and eBURST data, nine clades and singleton strains were identified. Clade 17 (13 strains, 28.3\%) and clade 1 ( 8 strains, $17.4 \%$ ) were the most and second most common, respectively (Figure 1 and Supplementary Table S1).

\section{Sequential Blood Strains Showed Evidence of Sequential Allelic Changes}

Five of the 37 patients had sequential strains isolated from their blood. Interestingly, three of these five patients were infected with more than one DST at the same time. Two patients had mixed infections with two DSTs from blood cultures collected on the same date (patient 12 and patient 34; Figure 1 and Supplementary Table S1). Furthermore, C. albicans isolates from patient 8 had three different DSTs identified from sequential blood cultures on days 0,3 , and 5 as DST1710, DST2924, and DST2527, respectively (Supplementary Table S1). Further analysis of diploid allele types (DATs) of the three sequential strains showed sequential changes of only one DAT at a time of the two later-collected strains (Table 1). Specifically, DAT7 of the VPS13 gene of the S29 strain collected on day 0 changed to DAT100 of the S30 strain collected on day 3. Then, DAT5 of the ADP1 gene of the S29 strain changed to DAT6 of the S1 strain collected on day 5. The profiles of the allelic genes from the patients in whom these 
TABLE 1 | Genetic positions of polymorphism on allelic genes of the sequential strains from the three patients.

\begin{tabular}{|c|c|c|c|c|c|c|c|c|c|c|c|c|c|c|}
\hline \multirow{2}{*}{$\begin{array}{l}\text { Patient } \\
\text { No. }\end{array}$} & \multirow{2}{*}{$\begin{array}{c}\text { Sample } \\
\text { No. }\end{array}$} & \multirow{2}{*}{$\begin{array}{l}\text { Collection } \\
\text { Date }\end{array}$} & \multirow[t]{2}{*}{ DSTs } & \multicolumn{9}{|c|}{ ADP1 } & \multirow{2}{*}{$\begin{array}{r}\text { SYA1 } \\
1623\end{array}$} & \multirow{2}{*}{$\begin{array}{r}\text { VPS13 } \\
2359\end{array}$} \\
\hline & & & & 815 & 820 & 826 & 889 & 905 & 946 & 985 & 1005 & 1012 & & \\
\hline \multirow[t]{3}{*}{8} & S29 & Day 0 & 1710 & $\mathrm{~A} / \mathrm{G}$ & & & & & & & & & & A \\
\hline & S30 & Day 3 & 2924 & $A / G$ & & & & & & & & & & $\mathrm{~A} / \mathrm{T}$ \\
\hline & S1 & Day 5 & 2527 & A & & & & & & & & & & $\mathrm{A} / \mathrm{T}$ \\
\hline \multirow[t]{2}{*}{12} & S36 & Day 0 & 2459 & & & & & & & & & & C & \\
\hline & S37 & Day 0 & 1902 & & & & & & & & & & $\mathrm{~A} / \mathrm{C}$ & \\
\hline \multirow[t]{2}{*}{34} & S43/S44 & Day 0 & 1627 & & C & C & A & A & $\mathrm{G}$ & $\mathrm{G}$ & $\mathrm{T}$ & C & & \\
\hline & S45 & Day 0 & 1705 & & $\mathrm{C} / \mathrm{T}$ & $\mathrm{C} / \mathrm{T}$ & $A / G$ & $\mathrm{~A} / \mathrm{G}$ & $A / G$ & $A / G$ & $\mathrm{~A} / \mathrm{T}$ & $\mathrm{C} / \mathrm{T}$ & & \\
\hline
\end{tabular}

different DSTs were detected (patients 8, 12, and 34) are shown in Table 1.

\section{Clade 17 Strains Showed Significantly Stronger Hemolytic Activity}

As the clade 17 predominance in C. albicans blood strains is uncommon, the virulence phenotype and antifungal susceptibility testing results of clade 17 and the other clades were determined and compared. Four virulence phenotypes were tested. First, the phospholipase zone values of the $C$. albicans strains ranged from 0.50 to 0.89 . All C. albicans strains were phospholipase producers (Table 2 and Supplementary Table S1). Most C. albicans strains showed very strong phospholipase activity (30 strains, 65.2\%). No weak phospholipase producer was observed. The mean phospholipase activity of all strains was 0.675 ( \pm 0.072$)$. Second, none of the C. albicans produced proteinase (Supplementary Table S1). Third, hemolytic activity was detected in all strains (Table 2 and Supplementary Table S1). The hemolytic zone values ranged from 0.50 to 0.95 . Most strains had positive activity (60.9\%). The mean hemolytic activity of the C. albicans strains was $0.666( \pm 0.089)$. Finally, most $C$. albicans strains $(54.3 \%)$ were highly able to form a biofilm layer on the bottom surface of each well (Table 2 and Supplementary Table S2). Association analysis using the chi-square test revealed that clade 17 strains showed significantly stronger hemolytic activity than non-clade 17 strains $(69.2 \%$ versus $37.5 \%$ and $24 \% ; p=0.025)$. There was no significant association between the MLST clades and other virulence phenotypes $(p>0.05)$ (Table 2).

\section{Clade 17 Showed No Significant Difference in Antifungal Susceptibility Pattern}

All strains were susceptible to anidulafungin, micafungin, and caspofungin, and wild type to amphotericin B. Only one isolate $(2.2 \%)$ was resistant to both voriconazole and fluconazole. The MIC50, MIC90, and geometric mean of all nine drugs are shown in Table 3. There was no significant correlation between clade and antifungal susceptibility. Further association analysis revealed that the clade 17 strains did not cause significantly higher case fatality rates than clade 1 and the other clades (75\% versus $42.9 \%$ and 55\%; $p=0.340$ ) (Supplementary Table S2).

\section{DISCUSSION}

In developing countries, candidemia case fatality rates of more than $50 \%$ have been reported, which is similar to

TABLE 2 | Number of strains in each MLST cluster in correlation to their phenotypic virulence factors.

\begin{tabular}{|c|c|c|c|c|c|c|c|c|c|c|c|c|}
\hline \multirow[b]{2}{*}{ Clades } & \multicolumn{3}{|c|}{ Biofilm formation (\%) } & \multicolumn{3}{|c|}{ Phospholipase activity (\%) } & \multicolumn{2}{|c|}{ Hemolytic activity (\%) } & \multirow[t]{2}{*}{ Total } & \multicolumn{2}{|c|}{ Patient outcome** (\%) } & \multirow[t]{2}{*}{ Total } \\
\hline & High & Low & None & Medium & Strong & Very strong & Positive & Strong positive & & Cure & Dead & \\
\hline 1 & $\begin{array}{l}4 \\
(50)\end{array}$ & $\begin{array}{c}1 \\
(12.5)\end{array}$ & $\begin{array}{c}3 \\
(37.5)\end{array}$ & $\begin{array}{c}8 \\
(100)\end{array}$ & $\begin{array}{c}2 \\
(25)\end{array}$ & $\begin{array}{c}6 \\
(75)\end{array}$ & $\begin{array}{c}5 \\
(62.5)\end{array}$ & $\begin{array}{c}3 \\
(37.5)\end{array}$ & $\begin{array}{c}8 \\
(100)\end{array}$ & $\begin{array}{c}4 \\
(57.1)\end{array}$ & $\begin{array}{c}3 \\
(42.9)\end{array}$ & $\begin{array}{c}7 \\
(100)\end{array}$ \\
\hline 17 & $\begin{array}{c}6 \\
(46.2)\end{array}$ & $\begin{array}{c}4 \\
(30.8)\end{array}$ & $\begin{array}{c}3 \\
(23)\end{array}$ & $\begin{array}{c}13 \\
(100)\end{array}$ & $\begin{array}{c}5 \\
(38.5)\end{array}$ & $\begin{array}{c}8 \\
(61.5)\end{array}$ & $\begin{array}{c}4 \\
(30.8)\end{array}$ & $\begin{array}{c}9 \\
(69.2)\end{array}$ & $\begin{array}{c}13 \\
(100)\end{array}$ & $\begin{array}{c}3 \\
(25)\end{array}$ & $\begin{array}{c}9 \\
(75)\end{array}$ & $\begin{array}{c}12 \\
(100)\end{array}$ \\
\hline Other* & $\begin{array}{c}15 \\
(60)\end{array}$ & $\begin{array}{c}8 \\
(32)\end{array}$ & $\begin{array}{c}2 \\
(8)\end{array}$ & $\begin{array}{c}25 \\
(100)\end{array}$ & $\begin{array}{c}6 \\
(12)\end{array}$ & $\begin{array}{c}16 \\
(24)\end{array}$ & $\begin{array}{c}19 \\
(76)\end{array}$ & $\begin{array}{c}6 \\
(24)\end{array}$ & $\begin{array}{c}25 \\
(100)\end{array}$ & $\begin{array}{c}9 \\
(45)\end{array}$ & $\begin{array}{c}11 \\
(55)\end{array}$ & $\begin{array}{c}20 \\
(100)\end{array}$ \\
\hline All strains & $\begin{array}{c}25 \\
(54.3)\end{array}$ & $\begin{array}{c}13 \\
(28.3)\end{array}$ & $\begin{array}{c}8 \\
(17.4)\end{array}$ & $\begin{array}{c}46 \\
(100)\end{array}$ & $\begin{array}{c}13 \\
(28.3)\end{array}$ & $\begin{array}{c}30 \\
(65.2)\end{array}$ & $\begin{array}{c}28 \\
(60.9)\end{array}$ & $\begin{array}{c}18 \\
(39.1)\end{array}$ & $\begin{array}{c}46 \\
(100)\end{array}$ & $\begin{array}{c}16 \\
(41)\end{array}$ & $\begin{array}{c}23 \\
(59)\end{array}$ & $\begin{array}{c}39 \\
(100)\end{array}$ \\
\hline$P$ value & & 0.335 & & & 0.503 & & & & - & & 40 & - \\
\hline
\end{tabular}

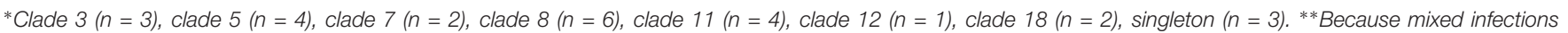

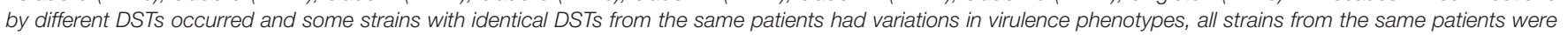
considered as different strains and analyzed separately. 
TABLE 3 | In vitro antifungal susceptibilities of $C$. albicans strains from hemoculturea, .

\begin{tabular}{|c|c|c|c|c|c|c|c|c|c|c|}
\hline \multirow[t]{2}{*}{ Drug } & \multicolumn{4}{|c|}{ MIC $(\mu / \mathrm{ml})$} & \multicolumn{4}{|c|}{ No. $(\%)$ of strains interpreted by CBPs } & \multicolumn{2}{|c|}{ No. $(\%)$ of strains interpreted by ECVs } \\
\hline & Range & MIC50 & MIC90 & GM & $\mathbf{s}$ & SDD & 1 & $\mathbf{R}$ & WT & Non-WT \\
\hline MFG & $\leq 0.008-0.015$ & $\leq 0.008$ & 0.008 & 0.008 & 46 (100\%) & $N / A$ & 0 & 0 & $46(100 \%)$ & 0 \\
\hline CFS & $0.008-0.12$ & 0.03 & 0.06 & 0.036 & $46(100 \%)$ & $N / A$ & 0 & 0 & $N / A$ & N/A \\
\hline$A m B$ & $0.25-0.5$ & 0.5 & 0.5 & 0.381 & $\mathrm{~N} / \mathrm{A}$ & $N / A$ & N/A & $\mathrm{N} / \mathrm{A}$ & $46(100 \%)$ & 0 \\
\hline VRC & $\leq 0.008-\geq 8$ & 0.008 & 0.015 & 0.010 & $45(97.83 \%)$ & 0 & $\mathrm{~N} / \mathrm{A}$ & 1 (2.17\%) & $\mathrm{N} / \mathrm{A}$ & N/A \\
\hline ITC & $0.03-\geq 16$ & 0.06 & 0.06 & 0.052 & $\mathrm{~N} / \mathrm{A}$ & $N / A$ & $\mathrm{~N} / \mathrm{A}$ & $\mathrm{N} / \mathrm{A}$ & N/A & N/A \\
\hline FLU & $0.12-16$ & 0.25 & 1 & 0.398 & 45 (97.83\%) & 0 & N/A & 1 (2.17\%) & N/A & N/A \\
\hline
\end{tabular}

${ }^{a} N / A$, not applicable; GM, geometric mean; CBP, clinical breakpoint; ECV, epidemiology cut-off value; S, susceptible; SDD, susceptible dose dependent; I, intermediate; R, resistant; WT, wild-type strain; AFG, anidulafungin; MFG, micafungin; CFG, caspofungin; AmB, amphotericin B; 5-FC, 5-flucytosine; PSO, posaconazole; VRC, voriconazole; ITC, itraconazole; FLU, fluconazolea. ${ }^{b}$ Interpretation (CBP and ECV: ug/ml) of susceptibility testing were in accordance with the recommendation of the CLSI for C. albicans; CBPs: Anidulafungin, Micafungin and Caspofungin: $S<25,1=0.5, R>1$; Voriconazole: $S<0.12, S D D=0.25-0.5, R>1$; Fluconazole: $S<2$, $S D D=4, R>8 ; E C V s$, Anidulafungin =0.12; Micafungin $=0.03 ;$ Amphotericin $B=2$.

the $54.8 \%$ case fatality rate found in this study (Kaur and Chakrabarti, 2017). Antibiotic resistance is generally one of the common causes of treatment failure of bloodstream infections that leads to patient fatality. However, such resistance is very rare for C. albicans blood strains. A comprehensive 2016 study of C. albicans bloodstream infections in East and Southeast Asia in found that just 1 out of 309 C. albicans blood strains resisted fluconazole, and all strains were sensitive to echinocandins (Tan et al., 2016). Similarly, C. albicans blood strains in our study were also highly susceptible to both echinocandins and azoles. This suggests that antifungal resistance is not a cause of treatment failure for C. albicans bloodstream infections. Rather, candidemia mortality is associated with patients' underlying conditions or management such as neutropenia from chemotherapy, septic shock, or ICU admission (Boonyasiri et al., 2013). However, comparisons of these factors for C. albicans and non-albicans Candida are lacking.

Candidemia risk factors are either healthcare related or host related (Kaur and Chakrabarti, 2017). In this study, almost all patients had both types of risk factors, specifically healthcare-related infections and immunocompromising conditions. In fact, none of the 32 patients whose clinical data were available had no risk factors. However, indepth details of the candidemia risk factors, including specific healthcare-related factors (such as use of a central vascular catheter, total parenteral nutrition, urinary catheter) and previous colonization of C. albicans (Kaur and Chakrabarti, 2017), were not available in our database. With the absence of primary sources regarding candidemia in Thailand, as evidenced by this study (Boonyasiri et al., 2013), determining C. albicans colonization is crucial, but it is not common practice in Thailand. Further collection and comprehensive analysis of such data would give an insight into specific risk factors for candidemia in Thailand.
High diversity among sequence types of C. albicans was reported in several previous studies (Odds et al., 2007; Shin et al., 2011; Alastruey-Izquierdo et al., 2013; Asadzadeh et al., 2017). Among 46 clinical strains in this study, high sequence variation was also observed with 34 distinct DSTs. DST254 was derived from the parental DST69, the most common DST found using MLST worldwide (Odds et al., 2007). However, DST69 was not found in this study. Additionally, DST443 and DST693, found in 6.5 and $2.2 \%$ of cases in this study, respectively, were the most prevalent DSTs in Taiwan (4.6 and 6.7\%, respectively) and China (DST443, 7.7\%) (Hu et al., 2015; Wang et al., 2015). DST443, DST457, and DST445 are particular sequence types found in Asia (Wang et al., 2015) that were also present in this study.

In general, clade 17 is more common in Asia (8.2\%) than other continents (0-6.2\%) (Odds et al., 2007). Moreover, a 2015 Chinese study also reported clade 17 as the second most common clade identified from $40 \mathrm{C}$. albicans non-blood strains ( $\mathrm{Wu}$ et al., 2015). However, when considering only blood strains, clade 17 was rare $(3.8 \%)$ in the globally collected C. albicans samples, and clade 1 was the most common (27.8\%) (Odds et al., 2007). Furthermore, no clade 17 strains were found in $149 \mathrm{C}$. albicans blood strains collected in a recent study from Korea (Jung et al., 2016). Only 10.9 and 15\% of C. albicans blood strains were identified as clade 17 in Taiwan (Wang et al., 2015) and China (Wu et al., 2015), respectively. Therefore, virulence studies were conducted to determine whether the Thai clade 17 isolates were more virulent than isolates from the other clades. The unusually high clade 17 prevalence of the Thai $C$. albicans blood strains might be explained by the stronger hemolytic activity that is required for C. albicans bloodstream infection (Furlaneto et al., 2018). However, clade 17 strains did not cause a significantly higher case fatality rate than the other strains. This suggests that many other factors also affect pathogenesis and patient mortality, especially host 
and environmental factors (Boonyasiri et al., 2013; Kaur and Chakrabarti, 2017).

Phospholipase enzyme plays an important role in host tissue invasion by disrupting the epithelial cell membrane. In general, C. albicans strains isolated from blood samples had higher enzyme activity than other anatomical sites (Ibrahim et al., 1995; Mohan Das and Ballal, 2008). For example, blood strains of C. albicans from Saudi Arabia (Fotedar and Al-Hedaithy, 2005), the European SENTRY program (Borst and Fluit, 2003), Brazil (Mattei et al., 2013), and Turkey (Atalay et al., 2015) showed 100, 71, 78, and 88\% positive phospholipase activity, respectively. Similarly, all C. albicans in this study showed positive phospholipase activity. However, despite their similar function regarding host invasion, unlike phospholipase, proteinase activity was typically undetectable in blood strains, whereas $95 \%$ of the strains from other sources had proteinase activity (Kantarcioglu and Yucel, 2002). In this study, proteinase activity was undetected in all C. albicans strains. Conversely, the European SENTRY program reported that approximately $80 \%$ of strains were positive for proteinase (Borst and Fluit, 2003). This suggests that proteinase activity variations might depend not only on the infection source but also on the strains' geographic location (De Bernardis et al., 2001).

Both hemolysin and biofilm formation are important virulence factors for C. albicans (Mayer et al., 2013). Hemolysin facilitates iron acquisition in disseminated candidiasis (Tsang et al., 2007), while biofilm formation protects the yeast from a harsh environment including nutrient storage, metabolic cooperation, and acquisition of new genetic traits (Mayer et al., 2013). In our study, C. albicans highly expressed both hemolytic activity (100\%) and biofilm formation (82.6\%). This suggests that both are important virulence factors for bloodstream infection.

Globally, C. albicans showed high susceptibility toward fluconazole, voriconazole, and echinocandins. Azole resistance was generally region dependent and uncommonly found in C. albicans (Lyon et al., 2010; Tan et al., 2016). All C. albicans strains in this study showed high susceptibility to anidulafungin, micafungin, and caspofungin, similar to previous studies from Australia, the Asia-Pacific, and Italy (Prigitano et al., 2016; Tan et al., 2016; Chapman et al., 2017). Only one (2.17\%) C. albicans isolate was resistant to voriconazole and fluconazole. This isolate was identified as DST2527, which resisted both voriconazole and fluconazole. This finding contrasts with another study from Taiwan (Wang et al., 2015). Therefore, association of DST2527 with resistance is unlikely. Moreover, when categorizing strains based on ECVs, all C. albicans strains also had wild-type MICs for amphotericin B, similar to previous studies (Petlum et al., 2013;

\section{REFERENCES}

Afsarian, M. H., Badali, H., Boekhout, T., Shokohi, T., and Katiraee, F. (2015). Multilocus sequence typing of Candida albicans isolates from a burn intensive care unit in Iran. J. Med. Microbiol. 64, 248-253. doi: 10.1099/jmm.0.0 00015

Alastruey-Izquierdo, A., Mandelblat, M., Ben Ami, R., Perlin, D. S., and Segal, E. (2013). Multilocus sequence typing of Candida albicans isolates from
Posteraro et al., 2015; Tan et al., 2016; Chapman et al., 2017). These data confirm echinocandins, fluconazole, and amphotericin B as the drugs of choice for treating patients with candidemia (Pappas et al., 2016). Moreover, a recent study showed that a combination of echinocandin and triazole drugs had potent activity for Candida spp., and triazole drugs had potent activity for Candida spp. (Fakhim et al., 2017). Therefore, antifungal testing for such a combination drug is beneficial, and its interpretation criteria should be established.

\section{CONCLUSION}

In conclusion, the molecular epidemiology among C. albicans blood strains in Thailand showed high genetic diversity. No significant association existed between patient fatality rate and the MLST clades. Clade 17 strains as a predominant clade among isolates showed significant association with hemolytic activity. This finding requires further strain sampling and pathogenetic study.

\section{ETHICS STATEMENT}

This study was approved by the Siriraj Institutional Review Board, No. SI 091/2016. Informed consent was not required in this work.

\section{AUTHOR CONTRIBUTIONS}

PN, PC, and KS designed the study. LP and SP performed the experiments and analyzed the data. LP, SP, and PN wrote the manuscript. All authors read and approved the manuscript.

\section{FUNDING}

The study was supported by the Siriraj Research Development Fund, grant number (IO) R016033021, Faculty of Medicine Siriraj Hospital, Mahidol University to PN, and Siriraj Graduate Scholarship to LP.

\section{SUPPLEMENTARY MATERIAL}

The Supplementary Material for this article can be found online at: https://www.frontiersin.org/articles/10.3389/fmicb. 2019.01194/full\#supplementary-material

candidemia and superficial candidiasis in Israel. Med. Mycol. 51, 755-758. doi: 10.3109/13693786.2013.777164

Asadzadeh, M., Ahmad, S., Al-Sweih, N., and Khan, Z. (2017). Molecular fingerprinting studies do not support intrahospital transmission of Candida albicans among candidemia patients in Kuwait. Front. Microbiol. 8:247. doi: 10.3389/fmicb.2017.00247

Atalay, M. A., Koc, A. N., Demir, G., and Sav, H. (2015). Investigation of possible virulence factors in Candida strains isolated from blood 
cultures. Niger. J. Clin. Pract. 18, 52-55. doi: 10.4103/1119-3077.14 6979

Boonyasiri, A., Jearanaisilavong, J., and Assanasen, S. (2013). Candidemia in Siriraj Hospital: epidemiology and factors associated with mortality. J. Med. Assoc. Thai. 96(Suppl. 2), S91-S97.

Borst, A., and Fluit, A. C. (2003). High levels of hydrolytic enzymes secreted by Candida albicans isolates involved in respiratory infections. J. Med. Microbiol. 52, 971-974. doi: 10.1099/jmm.0.05 228-0

Bougnoux, M. E., Diogo, D., Francois, N., Sendid, B., Veirmeire, S., Colombel, J. F., et al. (2006). Multilocus sequence typing reveals intrafamilial transmission and microevolutions of Candida albicans isolates from the human digestive tract. J. Clin. Microbiol. 44, 1810-1820. doi: 10.1128/jcm.44.5.1810-1820.2006

Bougnoux, M. E., Morand, S., and D'enfert, C. (2002). Usefulness of multilocus sequence typing for characterization of clinical isolates of Candida albicans. J. Clin. Microbiol. 40, 1290-1297. doi: 10.1128/jcm.40.4.1290-1297.2002

Bougnoux, M. E., Tavanti, A., Bouchier, C., Gow, N. A., Magnier, A., Davidson, A. D., et al. (2003). Collaborative consensus for optimized multilocus sequence typing of Candida albicans. J. Clin. Microbiol. 41, 5265-5266. doi: 10.1128/jcm. 41.11.5265-5266.2003

Bustamante, B., Martins, M. A., Bonfietti, L. X., Szeszs, M. W., Jacobs, J., Garcia, C., et al. (2014). Species distribution and antifungal susceptibility profile of Candida isolates from bloodstream infections in Lima, Peru. J. Med. Microbiol. 63, 855-860. doi: 10.1099/jmm.0.071167-0

Casadevall, A., and Pirofski, L. A. (1999). Host-pathogen interactions: redefining the basic concepts of virulence and pathogenicity. Infect. Immun. 67, 3703-3713.

Chapman, B., Slavin, M., Marriott, D., Halliday, C., Kidd, S., Arthur, I., et al. (2017). Changing epidemiology of candidaemia in Australia. J. Antimicrob. Chemother. 72, 1103-1108. doi: 10.1093/jac/dkw422

Clinical Laboratory Standards Institute (2012). Reference Method for Broth Dilution Antifungal Susceptibility Testing of Yeasts, 4th suppl., M27-S4. Wayne, PA: CLSI.

Clinical Laboratory Standards Institute (2016). Epidemiological Cutoff Values for Antifungal Susceptibility Testing, CLSI suppl M59 document. Wayne, PA: CLSI.

De Bernardis, F., Sullivan, P. A., and Cassone, A. (2001). Aspartyl proteinases of Candida albicans and their role in pathogenicity. Med. Mycol. 39, 303-313. doi: 10.1080/mmy.39.4.303.313

Diba, K., Makhdoomi, K., Nasri, E., Vaezi, A., Javidnia, J., Gharabagh, D. J., et al. (2018). Emerging Candida species isolated from renal transplant recipients: species distribution and susceptibility profiles. Microb. Pathog. 125, 240-245. doi: 10.1016/j.micpath.2018.09.026

Doi, A. M., Pignatari, A. C., Edmond, M. B., Marra, A. R., Camargo, L. F., Siqueira, R. A., et al. (2016). Epidemiology and microbiologic characterization of nosocomial candidemia from a Brazilian national surveillance program. PLoS One 11:e0146909. doi: 10.1371/journal.pone.0146909

Fakhim, H., Chowdhary, A., Prakash, A., Vaezi, A., Dannaoui, E., Meis, J. F., et al. (2017). In vitro interactions of echinocandins with triazoles against multidrug-resistant Candida auris. Antimicrob. Agents Chemother. 61:e1056-17. doi: 10.1128/AAC.01056-17

Fotedar, R., and Al-Hedaithy, S. S. (2005). Comparison of phospholipase and proteinase activity in Candida albicans and C. dubliniensis. Mycoses 48, 62-67. doi: 10.1111/j.1439-0507.2004.01057.x

Furlaneto, M. C., Goes, H. P., Perini, H. F., Dos Santos, R. C., and Furlaneto-Maia, L. (2018). How much do we know about hemolytic capability of pathogenic Candida species? Folia. Microbiol. 63, 405-412. doi: 10.1007/s12223-018-0 584-5

Gong, Y. B., Jin, B., Qi, H., Zhang, R., Zhang, X. Y., Yuan, P., et al. (2018). Multilocus sequence typing of Candida albicans isolates from the oral cavities of patients undergoing haemodialysis. Sci. Rep. 8:16413. doi: 10.1038/s41598018-34565-7

Hasan, F., Xess, I., Wang, X., Jain, N., and Fries, B. C. (2009). Biofilm formation in clinical Candida isolates and its association with virulence. Microbes Infect. 11, 753-761. doi: 10.1016/j.micinf.2009.04.018

Hu, L., Du, X., Li, T., Song, Y., Zai, S., Hu, X., et al. (2015). Genetic and phenotypic characterization of Candida albicans strains isolated from infectious disease patients in Shanghai. J. Med. Microbiol. 64, 74-83. doi: 10.1099/jmm.0.080 200-0
Ibrahim, A. S., Mirbod, F., Filler, S. G., Banno, Y., Cole, G. T., Kitajima, Y., et al. (1995). Evidence implicating phospholipase as a virulence factor of Candida albicans. Infect. Immun. 63, 1993-1998.

Jung, S. I., Shin, J. H., Kim, S. H., Kim, J., Kim, J. H., Choi, M. J., et al. (2016). Comparison of E,E-farnesol secretion and the clinical characteristics of Candida albicans bloodstream isolates from different multilocus sequence typing clades. PLoS One 11:e0148400. doi: 10.1371/journal.pone.014 8400

Kantarcioglu, A. S., and Yucel, A. (2002). Phospholipase and protease activities in clinical Candida isolates with reference to the sources of strains. Mycoses 45, 160-165. doi: 10.1046/j.1439-0507.2002.00727.x

Kaur, H., and Chakrabarti, A. (2017). Strategies to reduce mortality in adult and neonatal candidemia in developing countries. J. Fungi 3:41. doi: 10.3390/ jof3030041

Li, X., Yan, Z., and Xu, J. (2003). Quantitative variation of biofilms among strains in natural populations of Candida albicans. Microbiology 149, 353-362. doi: $10.1099 /$ mic. $0.25932-0$

Luo, G., Samaranayake, L. P., and Yau, J. Y. (2001). Candida species exhibit differential in vitro hemolytic activities. J. Clin. Microbiol. 39, 2971-2974. doi: $10.1128 / \mathrm{jcm} .39 .8 .2971-2974.2001$

Lyon, G. M., Karatela, S., Sunay, S., Adiri, Y., and Candida Surveillance Study Investigators. (2010). Antifungal susceptibility testing of Candida isolates from the Candida Surveillance study. J. Clin. Microbiol. 48, 1270-1275. doi: 10.1128/ JCM.02363-09

Macfarlane, M. G., and Knight, B. C. (1941). The biochemistry of bacterial toxins: the lecithinase activity of Cl. welchii toxins. Biochem. J. 35, 884-902. doi: $10.1042 / \mathrm{bj0350884}$

Mattei, A. S., Alves, S. H., Severo, C. B., Guazzelli Lda, S., Oliveira Fde, M., and Severo, L. C. (2013). Determination of germ tube, phospholipase, and proteinase production by bloodstream isolates of Candida albicans. Rev. Soc. Bras. Med. Trop. 46, 340-342. doi: 10.1590/0037-8682-0045-2013

Mayer, F. L., Wilson, D., and Hube, B. (2013). Candida albicans pathogenicity mechanisms. Virulence 4, 119-128. doi: 10.4161/viru.22913

Mohan Das, V., and Ballal, M. (2008). Proteinase and phospholipase activity as virulence factors in Candida species isolated from blood. Rev. Iberoam. Micol. $25,208-210$.

Odds, F. C., Bougnoux, M. E., Shaw, D. J., Bain, J. M., Davidson, A. D., Diogo, D., et al. (2007). Molecular phylogenetics of Candida albicans. Eukaryot. Cell 6, $1041-1052$.

Oz, Y., Dag, I., and Kiraz, N. (2012). Efficacy of disinfectants on Candida biofilms at different concentrations and contact times. Br. Microbiol. Res. J. 2, 40-52. doi: $10.9734 / \mathrm{bmrj} / 2012 / 1281$

Pappas, P. G., Kauffman, C. A., Andes, D. R., Clancy, C. J., Marr, K. A., OstroskyZeichner, L., et al. (2016). Clinical practice guideline for the management of candidiasis: 2016 update by the infectious diseases society of America. Clin. Infect. Dis. 62, e1-e50.

Petlum, P., Mongkol, N., Poosanga, P., Yowaphuy, M., and Foongladda, S. (2013). Candida species of bloodstream isolates and antifungal susceptibility of Candida in a tertiary hospital in Thailand. Siriraj. Med. J. 65(Suppl. 6), 20-23.

Posteraro, B., Spanu, T., Fiori, B., De Maio, F., De Carolis, E., Giaquinto, A., et al. (2015). Antifungal susceptibility profiles of bloodstream yeast isolates by Sensititre YeastOne over nine years at a large Italian teaching hospital. Antimicrob. Agents Chemother. 59, 3944-3955. doi: 10.1128/AAC.00285-15

Price, M. F., Wilkinson, I. D., and Gentry, L. O. (1982). Plate method for detection of phospholipase activity in Candida albicans. Sabouraudia 20, 7-14. doi: 10.1080/00362178285380031

Prigitano, A., Cavanna, C., Passera, M., Ossi, C., Sala, E., Lombardi, G., et al. (2016). CAND-LO 2014-15 study: changing epidemiology of candidemia in Lombardy (Italy). Infection 44, 765-780. doi: 10.1007/s15010-016-0951-6

Rossoni, R. D., Barbosa, J. O., Vilela, S. F., Jorge, A. O., and Junqueira, J. C. (2013). Comparison of the hemolytic activity between C. albicans and nonalbicans Candida species. Braz. Oral. Res. 27, 484-489. doi: 10.1590/S180683242013000600007

Sachin, C., Ruchi, K., and Santosh, S. (2012). In vitro evaluation of proteinase, phospholipase and haemolysin activities of Candida species isolated from clinical specimens. Int. J. Med. Biomed. Res. 1, 153-157. doi: 10.14194/ijmbr. 1211 
Shin, J. H., Bougnoux, M. E., D’enfert, C., Kim, S. H., Moon, C. J., Joo, M. Y., et al. (2011). Genetic diversity among Korean Candida albicans bloodstream isolates: assessment by multilocus sequence typing and restriction endonuclease analysis of genomic DNA by use of BssHII. J. Clin. Microbiol. 49, 2572-2577. doi: 10.1128/JCM.02153-10

Staib, F. (1965). Serum-proteins as nitrogen source for yeastlike fungi. Sabouraudia 4, 187-193. doi: 10.1080/00362176685190421

Tadec, L., Talarmin, J. P., Gastinne, T., Bretonniere, C., Miegeville, M., Le Pape, P., et al. (2016). Epidemiology, risk factor, species distribution, antifungal resistance and outcome of candidemia at a single French hospital: a 7-year study. Mycoses 59, 296-303. doi: 10.1111/myc. 12470

Tan, T. Y., Hsu, L. Y., Alejandria, M. M., Chaiwarith, R., Chinniah, T., Chayakulkeeree, M., et al. (2016). Antifungal susceptibility of invasive Candida bloodstream isolates from the Asia-Pacific region. Med. Mycol. 54, 471-477. doi: $10.1093 / \mathrm{mmy} / \mathrm{myv} 114$

Tavanti, A., Davidson, A. D., Fordyce, M. J., Gow, N. A., Maiden, M. C., and Odds, F. C. (2005). Population structure and properties of Candida albicans, as determined by multilocus sequence typing. J. Clin. Microbiol. 43, 5601-5613. doi: $10.1128 / \mathrm{jcm} .43 .11 .5601-5613.2005$

Tsai, M. H., Wang, S. H., Hsu, J. F., Lin, L. C., Chu, S. M., Huang, H. R., et al. (2015). Clinical and molecular characteristics of bloodstream infections caused by Candida albicans in children from 2003 to 2011. Clin. Microbiol. Infect. 21:1018.e1-8. doi: 10.1016/j.cmi.2015.06.024

Tsang, C. S., Chu, F. C., Leung, W. K., Jin, L. J., Samaranayake, L. P., and Siu, S. C. (2007). Phospholipase, proteinase and haemolytic activities of Candida albicans isolated from oral cavities of patients with type 2 diabetes mellitus. J. Med. Microbiol. 56, 1393-1398. doi: 10.1099/jmm.0.47303-0

Vaezi, A., Fakhim, H., Khodavaisy, S., Alizadeh, A., Nazeri, M., Soleimani, A., et al. (2017). Epidemiological and mycological characteristics of candidemia in Iran: a systematic review and meta-analysis. J. Mycol. Med. 27, 146-152. doi: 10.1016/j.mycmed.2017.02.007

Wang, S. H., Shen, M., Lin, H. C., Sun, P. L., Lo, H. J., and Lu, J. J. (2015). Molecular epidemiology of invasive Candida albicans at a tertiary hospital in northern
Taiwan from 2003 to 2011. Med. Mycol. 53, 828-836. doi: 10.1093/mmy/ myv065

Wiederhold, N. P. (2017). Antifungal resistance: current trends and future strategies to combat. Infect. Drug Resist. 10, 249-259. doi: 10.2147/IDR.S124918

Wu, J. Y., Guo, H., Wang, H. M., Yi, G. H., Zhou, L. M., He, X. W., et al. (2017). Multilocus sequence analyses reveal extensive diversity and multiple origins of fluconazole resistance in Candida tropicalis from tropical China. Sci. Rep. 7:42537. doi: 10.1038/srep42537

Wu, K., Luo, T., Li, L., Zhang, Q., Zhu, J., Gao, Q., et al. (2015). multilocus sequence typing of pathogenic candida albicans isolates collected from a teaching hospital in Shanghai, China: a molecular epidemiology study. PLoS One 10:e125245. doi: 10.1371/journal.pone.0125245

Yapar, N. (2014). Epidemiology and risk factors for invasive candidiasis. Ther. Clin. Risk Manag. 10, 95-105. doi: 10.2147/TCRM.S40160

Yazdanparast, S. A., Khodavaisy, S., Fakhim, H., Shokohi, T., Haghani, I., Nabili, M., et al. (2015). Molecular characterization of highly susceptible Candida africana from vulvovaginal candidiasis. Mycopathologia 180, 317-323. doi: 10.1007/s11046-015-9924-Z

Yigit, N., Aktas, E., Dagistan, S., and Ayyildiz, A. (2011). Investigating biofilm production, coagulase and hemolytic activity in Candida species isolated from denture stomatitis patients. Eurasian J. Med. 43, 27-32. doi: 10.5152/eajm. 2011.06

Conflict of Interest Statement: The authors declare that the research was conducted in the absence of any commercial or financial relationships that could be construed as a potential conflict of interest.

Copyright (c) 2019 Pham, Pharkjaksu, Chongtrakool, Suwannakarn and Ngamskulrungroj. This is an open-access article distributed under the terms of the Creative Commons Attribution License (CC BY). The use, distribution or reproduction in other forums is permitted, provided the original author(s) and the copyright owner(s) are credited and that the original publication in this journal is cited, in accordance with accepted academic practice. No use, distribution or reproduction is permitted which does not comply with these terms. 